\title{
General Relativistic Orbital Effects in Compact Binary Stars (Solution by the Method of Celestial Mechanics)
}

\author{
Linsen Li \\ School of Physics, Northeast Normal University, Changchun, China \\ Email: dbsd-1ls@163.com
}

How to cite this paper: Li, L.S. (2017) General Relativistic Orbital Effects in Compact Binary Stars (Solution by the Method of Celestial Mechanics). World Journal of Mechanics, 7, 360-369.

https://doi.org/10.4236/wjm.2017.712027

Received: September 25, 2017

Accepted: December 26, 2017

Published: December 29, 2017

Copyright $\odot 2017$ by author and Scientific Research Publishing Inc. This work is licensed under the Creative Commons Attribution International License (CC BY 4.0).

http://creativecommons.org/licenses/by/4.0/

\begin{abstract}
Perturbation methods are employed to calculate time variation in the orbital elements of a compact binary system. It turns out that the semi-major axis and eccentricity exhibit only periodic variations. The longitude of periastron and mean longitude of epoch exhibit both secular and periodic variation. In addition, the relativistic effects on the time of periastron passage of binary stars are also given. Four compact binary systems (PSRJ0737-3039, PSR1913+16, PSR1543+12 and M33X-7) are considered. Numerical results for both secular and periodic effects are presented, and the possibility of observing them is discussed.
\end{abstract}

\section{Keywords}

General Relativistic Orbital Effect, Compact Binary Stars

\section{Introduction}

In the wake of unceasing development in the post-Newtonian celestial mechanics, at present, the research on the post-Newtonian effects has been exhibited gradually due to the fact that the degree of accuracy of astronomical observation improves unceasingly. Hence several authors devoted their research to this subject and the scopes (Brumberg, $(1972,1985)$ [1] [2], Rubincam (1977) [3], Soffel $(1987,1989)$ [4] [5], Iorio (2005) [6]). These authors research mainly the postNewtonian effect of the orbital elements of planets and artificial satellites in the solar system. The largest post-Newtonian effects have been exhibited in the orbits of binary systems, especially in the compact binary systems. So, the research in the post-Newtonian orbital effect of the compact binary stars is important and 
meaningful. Hence some authors research this subject from theory and observation. Such as, Will $(1981,2006)$ [7] [8], Damour \& Deruelle $(1985,1986)$ [9] [10], Schāfer \& Wex (1993) [11], Wex (1995) [12]. Calura (1997) [13], Iorio (2007) [14] studied this subject from theory. On the other hand, Burgay et al. (2003) [15], Konacki (2003) [16], Kramer et al. (2005) [17], and Weisberg \& Taylor (2005) [18] researched the post-Newtonian effect for the periastron shift of compact binary stars (PSRJ0737-3039, PSR1913+16 PSR1543+12) from observation. These researches are very interested in the theoretical and observing aspects. This paper presents the post-Newtonian effect on all orbital elements of the compact binary stars on the theoretical aspect. The research method of this paper is different from previous studies that this paper uses the method of perturbation theory in the celestial mechanics.

\section{R, S and W Components for the General Relativistic Accelerations in the Two-Body Problem}

The relative acceleration of two-body with the Post-Newtonian Parameters is given by Will (1981) [7]

$$
\begin{aligned}
\boldsymbol{a}_{P N}= & \frac{m \boldsymbol{X}}{r^{3}}\left[(2 \gamma+2 \beta) \frac{m}{r}-\gamma v^{2}+\left(2+\alpha_{1}-2 \zeta_{2}\right) \frac{\mu}{r}\right. \\
& \left.-\frac{1}{2}\left(6+\alpha_{1}+\alpha_{2}+\alpha_{3}\right) \frac{\mu}{m} v^{2}+\frac{3}{2}\left(1+\alpha_{2}\right) \frac{\mu}{m}(\boldsymbol{v} \cdot \boldsymbol{n})^{2}\right] \\
& +\frac{m(\boldsymbol{X} \cdot \boldsymbol{v}) \bar{v}}{r^{3}}\left[(2 \gamma+2)-\frac{\mu}{m}\left(2-\alpha_{1}+\alpha_{2}\right)\right] .
\end{aligned}
$$

here $\quad m=m_{1}+m_{2}, \quad \mu=\frac{m_{1} m_{2}}{m}, \quad \boldsymbol{n}=\frac{\boldsymbol{X}}{r}, \quad \boldsymbol{X}=\boldsymbol{n} \cdot r=\boldsymbol{r}, \quad \boldsymbol{r} \cdot \dot{\boldsymbol{i}}=r \cdot \dot{r}$, $\boldsymbol{v}=\dot{\boldsymbol{r}}=\dot{\boldsymbol{r}} \boldsymbol{n}+\dot{r} \dot{f} \lambda, \quad v^{2}=\dot{\boldsymbol{r}}^{2}+r^{2} \dot{f}^{2}, \quad(\boldsymbol{v} \cdot \boldsymbol{n})^{2}=\left(\overline{\boldsymbol{v}} \cdot \frac{\boldsymbol{X}}{r}\right)^{2}=\frac{(\boldsymbol{v} \cdot \boldsymbol{n} \boldsymbol{r})^{2}}{r^{2}}=\frac{\left(\dot{\boldsymbol{r}}^{2} \cdot \boldsymbol{r}\right)^{2}}{r^{2}}=\dot{\boldsymbol{r}}^{2}$

$$
\begin{aligned}
\frac{m(\boldsymbol{X} \cdot \boldsymbol{v}) \boldsymbol{v}}{r^{3}} & =\frac{m}{r^{3}}(\boldsymbol{n} r \cdot \boldsymbol{v}) \boldsymbol{v} \\
& =\frac{m}{r^{3}}(\boldsymbol{r} \cdot \dot{\boldsymbol{r}}) \dot{\boldsymbol{r}} \\
& =\frac{m}{r^{3}}(r \cdot \dot{r})(\dot{\boldsymbol{r}} \boldsymbol{n}+r \dot{r} \lambda) \\
& =\frac{m}{r^{3}}\left(r \dot{r} \boldsymbol{n}+r^{2} \dot{\boldsymbol{r}} \dot{f} \lambda\right) .
\end{aligned}
$$

here $f$ denotes the true anomaly. $\boldsymbol{n}$ is a unit vector in the radial direction and $\lambda$ are unit vectors in the orbital plane. $n$ is directed along the radial direction, and $\lambda$ is perpendicular to $\boldsymbol{n}$. In the equation $\mathrm{m}$ denotes $G \mathrm{~m}$ and the right side should be multiplied by $c^{-2}$. $G$ is gravitational constant and $c$ is the speed of light.

In this paper we research the general relativistic effect. In the general relativistic case the Post-Newtonian parameters $\alpha_{1}=\alpha_{2}=\alpha_{3}=0, \quad \beta=1, \quad \gamma=1$, $\zeta_{2}=0$. (Will 1981) [7]. The Equation (1) can be written 


$$
\begin{aligned}
\boldsymbol{a}_{P N}= & \frac{m \boldsymbol{X}}{r^{3}}\left[4 \frac{m}{r}-v^{2}+2 \frac{\mu}{r}-3 \frac{\mu}{m} v^{2}+\frac{3}{2} \frac{\mu}{m}(\boldsymbol{v} \cdot \boldsymbol{n})^{2}\right] \\
& +\frac{m(\boldsymbol{X} \cdot \boldsymbol{v}) \bar{v}}{r^{3}}\left[4-2 \frac{\mu}{m}\right] .
\end{aligned}
$$

Using the relative expressions the Equation (3) may be written

$$
\begin{aligned}
\boldsymbol{a}_{p p n}= & \frac{m}{r^{3}}(r \boldsymbol{n})\left[4 \frac{m}{r}+2 \frac{\mu}{r}-\left\{1+3 \frac{\mu}{m}\right)\left(\dot{r}^{2}+r^{2} \dot{f}^{2}\right)+\frac{3}{2} \frac{\mu}{m} \dot{r}^{2}\right] \\
& +\frac{m}{r^{3}}\left[\left(r \dot{r}^{2} \boldsymbol{n}+r^{2} \dot{r} \dot{f} \lambda\right)\left\{4-2 \frac{\mu}{m}\right\}\right] .
\end{aligned}
$$

here boldface denotes vector.

We resolve the acceleration $\boldsymbol{a}$ into a radial component $R \boldsymbol{n}$, a component $S \lambda$, normal to $R \boldsymbol{n}$ and a component $W$ normal to the orbital plane.

i.e, $\quad \boldsymbol{a}=R \boldsymbol{n}+S \lambda+W(\boldsymbol{n} \times \boldsymbol{\lambda}), \quad \boldsymbol{n} \times \boldsymbol{\lambda}=N$ (the unit vector normal to the orbital plane).

On comparison with the expression (4), we get three scalar accelerative components $R, S$ and $W$

$$
\begin{aligned}
R= & \frac{m}{r^{2}}\left[4 \frac{m}{r}-2 \frac{\mu}{r}-\left(1+3 \frac{\mu}{m}\right)\left(r^{\prime 2}+r^{2} \dot{f}^{2}\right)+\frac{3}{2} \dot{r}^{2} \frac{\mu}{m}\right] \\
& +\frac{m}{r^{2}} \dot{r}^{2}\left[4-2 \frac{\mu}{m}\right] \\
S= & \frac{m}{r}\left[4-2 \frac{\mu}{m}\right] \dot{r} \dot{f} \\
W= & 0
\end{aligned}
$$

Substituting the following formulas of the problem of two body into the above formula (Smart, 1953) [19]

$$
\dot{f}=\frac{\mathrm{d} f}{\mathrm{~d} t}=n a \sqrt[2]{1-e^{2}} / r^{2}, \dot{r}=\frac{n a e \sin f}{\sqrt{1-e^{2}}}, n^{2} a^{3}=m .
$$

We obtain

$$
\begin{aligned}
& r^{2} R=m^{2}\left(\frac{\left(4+2 \frac{\mu}{m}\right)}{r}+\left[3-\frac{7}{2} \frac{\mu}{m}\right] \frac{e^{2} \sin ^{2} f}{p}-\left(1+3 \frac{\mu}{m}\right] \frac{p}{r^{2}}\right], \\
& r^{2} S=\frac{m^{2}}{r}\left[4-2 \frac{\mu}{m}\right] e \sin f, \\
& W=0 .
\end{aligned}
$$

here $p=a\left(1-e^{2}\right)$.

An independent variable $\mathrm{d} t$ is transformed to an independent variable $\mathrm{d} f$ in the Gaussian perturbation equations (Brouwer \& Clemence, 1961 [20], Roy, 
1988) [21] by using the second formula of the Equations (6), we get the perturbation equations with an independent variable true anomaly $f$

$$
\left.\begin{array}{l}
\frac{\mathrm{d} a}{\mathrm{~d} f}=\frac{2 a}{m\left(1-e^{2}\right)}\left[R r^{2} e \sin f+S r^{2}\left(\frac{p}{r}\right)\right], \\
\frac{\mathrm{d} e}{\mathrm{~d} f}=\frac{1}{n^{2} a^{3}}\left[R r^{2} \sin f+S r^{2}(\cos E+\cos f)\right], \\
\frac{\mathrm{d} \tilde{\omega}}{\mathrm{d} f}=\frac{1}{n a e}\left[-R r^{2} \cos f+S r^{2}\left(1+\frac{r}{p}\right) \sin f\right], \\
\frac{\mathrm{d} \varepsilon_{0}}{\mathrm{~d} f}=-\frac{2 r^{3} R}{n^{2} a^{4} \sqrt{1-e^{2}}}+\frac{e^{2}}{1+\sqrt{1-e^{2}}} \frac{\mathrm{d} \omega}{\mathrm{d} f}, \\
\frac{\mathrm{d} i}{\mathrm{~d} f}=\frac{r^{3} \cos (\omega+f)}{n^{2} a^{4}\left(1-e^{2}\right)} W, \\
\frac{\mathrm{d} \Omega}{\mathrm{d} f}=\frac{r^{3} \sin (\omega+f)}{n^{2} a^{4}\left(1-e^{2}\right)} W,
\end{array}\right\}
$$

where $\tilde{\omega}$ is the longitude of periastron, $\mathrm{E}$ is the eccentric anomaly and $\varepsilon_{0}$ is the mean longitude at epoch.

\section{Integration for the Perturbation Equations and Its Perturbation Solutions}

Substituting R, S and W for expressions (7) into the perturbation Equation (8) by using Kepler's third law $n^{2} a^{3}=\left(m_{1}+m_{2}\right)=m$, we obtain

$$
\begin{aligned}
& \frac{\mathrm{d} a}{\mathrm{~d} f}=\frac{2 m}{\left(1-e^{2}\right)^{2}}\left\{\left[\left(7-3 \frac{\mu}{m}\right) e+\left(3-\frac{31 \mu}{8 m}\right) e^{3}\right] \sin f+\left(5-4 \frac{\mu}{m}\right) e^{2} \sin 2 f-\frac{3}{8} \frac{\mu}{m} e^{3} \sin 3 f\right\} \\
& \frac{\mathrm{d} e}{\mathrm{~d} f}=\frac{m}{p}\left\{\left[\left(3-\frac{\mu}{m}\right)+\left(7-\frac{47}{8} \frac{\mu}{m}\right) e^{2}\right] \sin f+\left(5-4 \frac{\mu}{m}\right) e \sin 2 f-\frac{3}{8} e^{2} \frac{\mu}{m} \sin 3 f\right\}, \\
& \frac{\mathrm{d} \tilde{\omega}}{\mathrm{d} f}=\frac{m}{e p}\left\{3 e+\left[\left(\frac{\mu}{m}-3\right)+\left(1+\frac{21}{8} \frac{\mu}{m}\right) e^{2}\right] \cos f-\left(5-4 \frac{\mu}{m}\right) e \cos 2 f+\frac{3}{8} \frac{\mu}{m} e^{2} \cos 3 f\right\}, \\
& \frac{\mathrm{d} i}{\mathrm{~d} f}=\frac{\mathrm{d} \Omega}{\mathrm{d} f}=0, \\
& \frac{\mathrm{d} \varepsilon_{0}}{\mathrm{~d} f}=-\frac{2 m}{a \sqrt{1-e^{2}}}\left\{\left(6-\frac{9}{2} \frac{\mu}{m}\right)+\left(3-\frac{7}{2} \frac{\mu}{m}\right) \frac{e^{2}-1}{p} r-\left(4-\frac{1}{2} \frac{\mu}{m}\right) e \cos f\right\} \\
& +\frac{e^{2}}{1+\sqrt{1-e^{2}}} \frac{\mathrm{d} \omega}{\mathrm{d} f} .
\end{aligned}
$$

Integrating the above Equation (9), we obtain the perturbation secular and periodic solutions 


$$
\begin{aligned}
\delta a= & -\frac{2 m}{\left(1-e^{2}\right)^{2}}\left\{\left[\left(7-3 \frac{\mu}{m}\right) e+\left(3-\frac{31}{8} \frac{\mu}{m}\right) e^{3}\right]\left(\cos f-\cos f_{0}\right)\right. \\
& \left.+\frac{1}{2}\left(5-4 \frac{\mu}{m}\right) e^{2}\left(\cos 2 f-\cos 2 f_{0}\right)-\frac{1}{8} \frac{\mu}{m} e^{3}\left(\cos 3 f-\cos 3 f_{0}\right)\right\}, \\
\delta e= & -\frac{m}{a\left(1-e^{2}\right)}\left\{\left[\left(3-\frac{\mu}{m}\right)+\left(7-\frac{47}{8} \frac{\mu}{m}\right) e^{2}\right]\left(\cos f-\cos f_{0}\right)\right. \\
& \left.+\frac{1}{2}\left(5-4 \frac{\mu}{m}\right) e\left(\cos 2 f-\cos 2 f_{0}\right)-\frac{1}{8} e^{2} \frac{\mu}{m}\left(\cos 3 f-\cos 3 f_{0}\right)\right\}, \\
\delta \tilde{\omega}= & \frac{m}{a\left(1-e^{2}\right) e}\left\{3 e\left(f-f_{0}\right)+\left[\left(\frac{\mu}{m}-3\right)+\left(1+\frac{21}{8} \frac{\mu}{m}\right) e^{2}\right]\left(\sin f-\sin f_{0}\right)\right\} \\
& \left.-\frac{1}{2}\left(5-4 \frac{\mu}{m}\right) e\left(\sin 2 f-\sin 2 f_{0}\right)+\frac{1}{8} \frac{\mu}{m} e^{2}\left(\sin 3 f-\sin 3 f_{0}\right)\right\}, \\
\delta \varepsilon_{0}= & -\frac{m}{a \sqrt{1-e^{2}}}\left\{\left(12-9 \frac{\mu}{m}\right)\left(f-f_{0}\right)-\left(6-7 \frac{\mu}{m}\right) \sqrt{1-e^{2}}\left(E-E_{0}\right)\right. \\
& \left.-\left(8-\frac{\mu}{m}\right) e\left(\sin f-\sin f_{0}\right)\right\}+\frac{e^{2}}{1+\sqrt{1-e^{2}}} \delta \omega, \\
\delta \lambda= & n\left(t-t_{0}\right)+\delta \varepsilon_{0}, \\
\delta i= & \delta \Omega=0, m=m_{1}+m_{2}
\end{aligned}
$$

where $\lambda$ denotes the mean longitude of periastron, $E$ denotes the eccentric anomaly. In the last integral expression, we have used the next integral already:

$$
\begin{aligned}
& \int r \mathrm{~d} f=a \int \sqrt{1-e^{2}} \mathrm{~d} E \\
& \int \frac{e^{2} \sin ^{2} f}{p} r \mathrm{~d} f=\frac{e^{2}-1}{p} \int r \mathrm{~d} f+\int \mathrm{d} f-e \int \cos f \mathrm{~d} f .
\end{aligned}
$$

\section{The Secular and Periodic Variation of the Orbital Elements}

1) The secular variation per cycle (revolution)

By letting $f_{0}=0, f=2 \pi, E_{0}=0, E=2 \pi$, the periodic terms are disappeared and one obtains the secular variables per cycle (revolution):

$$
\begin{aligned}
& \Delta a=0 \quad(\mathrm{~cm} / \text { cycle }) \\
& \Delta e=0 \quad(\text { /cycle }) \\
& \Delta \tilde{\omega}=6 \pi \frac{m}{a\left(1-e^{2}\right)} \quad(\mathrm{rad} / \text { cycle }) \\
& \Delta \varepsilon_{0}=-\left\{\frac{2 \pi m}{a \sqrt{1-e^{2}}}\left[\left(12-9 \frac{\mu}{m}\right)-\left(6-7 \frac{\mu}{m}\right)\left(1-e^{2}\right)^{1 / 2}\right]\right\} \\
& \Delta \lambda=\left(2 \pi+\Delta \varepsilon_{0}\right) \quad(\mathrm{rad} / \text { cycle }) \\
& \Delta i=\Delta \Omega=0 \quad(1 \mathrm{rad} / \text { cycle })
\end{aligned}
$$


where $\mu=G(M+m)$

The author Li (2010) [24] obtained the formulas for the post-Newtonian effect on the time variation of periastron passage of binary stars. In that paper we change the symbol of the first expression of (35) (in the case of relativity) as the symbol of the present paper, which is

$$
\Delta \tau=2 \pi a^{1 / 2} m^{1 / 2}\left[\left(9-2 \frac{\mu}{m}\right)-\frac{1}{2}\left(7-17 \frac{\mu}{m}\right) e+\left(51 / 2-24 \frac{\mu}{m}\right) e^{2}\right](\mathrm{s} / \mathrm{Rev}) .
$$

2) The secular variable rate:

$$
\left.\begin{array}{l}
\dot{a}=\dot{e}=\dot{i}=\dot{\Omega}=0 \\
\dot{\tilde{\omega}}=\Delta \tilde{\omega} / P(\mathrm{rad} / \mathrm{yr}) \\
\dot{\varepsilon}_{0}=\Delta \varepsilon_{0} / P(\mathrm{rad} / \mathrm{yr}) \\
\dot{\lambda}=\left[2 \pi / P+\Delta \varepsilon_{0} / P\right](\mathrm{rad} / \mathrm{yr}) \\
\dot{\tau}=\Delta \tau / P(\mathrm{~s} / \mathrm{d})
\end{array}\right\}
$$

where the period $P$ is denoted in unit of day

3) The periodic variation of amplitudes:

In the expressions (10) all terms are the periodic variable terms except for all secular terms. Here we list the maximal and minimal amplitudes of the periodic terms for semi-major axis, $a$ and eccentricity, $e$ from the expressions (10).

For the semi-major axis:

$$
\left.\begin{array}{l}
A_{\max }=-\frac{2 m}{\left(1-e^{2}\right)^{2}}\left[\left(7-3 \frac{\mu}{m}\right) e+\left(3-\frac{31}{8} \frac{\mu}{m}\right) e^{3}\right] ; \\
A_{\min }=+\frac{m}{4\left(1-e^{2}\right)^{2}} \frac{\mu}{m} e^{3}
\end{array}\right\}
$$

For eccentricity:

$$
\left.\begin{array}{l}
E_{\max }=-\frac{m}{a\left(1-e^{2}\right)}\left[\left(3-\frac{\mu}{m}\right)+\left(7-\frac{47}{8} \frac{\mu}{m}\right) e^{2}\right] ; \\
E_{\min }=+\frac{m}{8 a\left(1-e^{2}\right)} \frac{\mu}{m} e^{2}
\end{array}\right\}
$$

\section{Numerical Results for Four Compact Binary Systems}

We use the formulae (11) - (13) to calculate the secular of the general relativistic secular effect on the orbital elements of four compact binary systems. It is convenient to reduce the formulas (11) - (13) to practical units $m_{1}, m_{2}$ and $a$ are denoted by the unit in solar mass $M_{1}\left(m_{\odot}\right), M_{2}\left(m_{\odot}\right)$ and solar radius $A\left(R_{\odot}\right)$, and $\mathrm{P}$ is denoted by the unit in day $=86400 \mathrm{~s}, G=6.67 \times 10^{-8}$, (c. g. s), c $=3 \times$ $10^{10} \mathrm{~cm} / \mathrm{s}$. The formulae (11) - (13) can be written by taking the secular effect 


$$
\begin{aligned}
\Delta \tilde{\omega}= & 6 K \frac{\left(M_{1}+M_{2}\right)}{A\left(1-e^{2}\right)}(\mathrm{rad} / \text { cycle }) \\
\Delta \varepsilon_{0}= & -\left\{\frac{2 K\left(M_{1}+M_{2}\right)}{A \sqrt{1-e^{2}}}\left[\left(12-9 \frac{\mu}{m}\right)-\left(6-7 \frac{\mu}{m}\right)\left(1-e^{2}\right)^{1 / 2}\right]\right. \\
& \left.-\frac{6 K\left(M_{1}+M_{2}\right)}{A\left(1-e^{2}\right)}\left(\frac{e^{2}}{1+\sqrt{1-e^{2}}}\right)\right\}(\mathrm{rad} / \text { cycle }) \\
\Delta \lambda= & \left(2 \pi+\Delta \varepsilon_{0}\right)(\mathrm{rad} / \mathrm{cycle}) \\
\Delta i= & \Delta \Omega=0(\mathrm{rad} / \mathrm{cycle}) \\
\Delta \tau= & 2 A^{1 / 2}\left(M_{1}+M_{2}\right)^{1 / 2}\left[\left(9-2\left(\frac{\mu}{M+M_{2}}\right)-\frac{1}{2}\left(7-17 \frac{\mu}{M_{1}+M_{2}}\right) e\right.\right. \\
& \left.+\left(51 / 2-24 \frac{\mu}{M_{1}+M_{2}}\right) e^{2}\right](\mathrm{s} / \mathrm{Rev}) .
\end{aligned}
$$

here $K=\pi G M_{\odot} / c^{2} R_{\odot}=6.66 \times 10^{6} \quad(\mathrm{c}, \mathrm{g}, \mathrm{s})$.,

This paper chooses four compact binary systems: PSR1913+16, PSR1543+12, PSRJ0737-3039 and a black hole M33 X-7 as an example. For these compact binary stars, their data for P, a, e, M and m are retrieved from Burgay et al. (2003)

\begin{tabular}{|c|c|c|c|c|c|c|}
\hline $\begin{array}{c}\text { Compact } \\
\text { binary stars }\end{array}$ & $\mathrm{P}(\mathrm{d})$ & $\mathrm{A}\left(\mathrm{R}_{\odot}\right)$ & $\mathrm{e}$ & $\mathrm{M}_{1}(\mathrm{~m} \odot)$ & $\mathrm{M}_{2}\left(\mathrm{~m}_{\odot}\right)$ & Ref \\
\hline M33 x-7 & 3.450 & 42.40 & 0.0385 & 15.65 & 70.00 & Orosz et al. (2007) [23] \\
\hline PSR J0737-3039 & 0.1022 & 1.26 & 0.0878 & 1.34 & 1.25 & $\begin{array}{l}\text { Willems et al. (2004) [22] } \\
\text { Burgay et al. (2003) [15] } \\
\text { Kramar et al. (2005) [17] }\end{array}$ \\
\hline PSR1913+16 & 0.3230 & 2.80 & 0.6170 & 1.44 & 1.38 & Willems et al. (2004) [22] \\
\hline PSR1543+12 & 0.1022 & 3.28 & 0.2736 & 1.35 & 1.33 & $\begin{array}{l}\text { Konacki et al. (2003) [16] } \\
\text { Willems et al. (2004) [22] }\end{array}$ \\
\hline
\end{tabular}
[15], Konacki et al. (2003) [16], Kramer et al. (2005) [17], Willems et al. (2004) [22] and Orosz et al. (2007) [23]. Their data are listed in Table 1.

Substituting these data in Table 1 into formulas (16) and (13) and (14) - (15), we obtain the numerical results for the periodic and secular variation of the orbital elements of four compact binary stars in Tables 2-4.

Table 1. The data of four compact binary stars.

Table 2. Periodic variation of the amplitudes of the orbits of semi-major axis and eccentricity.

\begin{tabular}{ccccc}
\hline \multirow{2}{*}{$\begin{array}{c}\text { Compact } \\
\text { binary stars }\end{array}$} & \multicolumn{2}{c}{ Semi-major axis } & \multicolumn{2}{c}{ Eccentricity } \\
\cline { 2 - 5 } & $A_{\max }(\mathrm{km})$ & $A_{\min }(\mathrm{km})$ & $E_{\max }\left(\times 10^{-5}\right)$ & $E_{\min }\left(\times 10^{-6}\right)$ \\
\hline M33 X-7 & -1661.80 & 0.00027 & -1.23 & 0.00012 \\
PSR J0737-3039 & -48.55 & 0.00016 & -1.23 & 0.00100 \\
PSR1913+16 & -51.77 & 0.0560 & -1.68 & 0.0410 \\
PSR1543+12 & -60.83 & 0.0037 & -0.62 & 0.00270 \\
\hline
\end{tabular}


It can be seen from Table 2 that the maximum amplitude of semi-major axis is the black hole binary system M33 X-7 and the maximum eccentricity is PSR $1913+16$.

It can be seen from Table 3 that the maximum secular variable of longitude per period is PSRJO737-3039. The longest time of the periastron passage is M33-7.

It can be seen from Table 4 that the maximum secular variable rates of longitude is PSRJ0737-3039. The longest time of the periastron passage is M33X-7.

\section{Discussion and Conclusions}

1) The comparison of the theoretical results with the observable results.

The theoretical results in this paper as compared with the observable results given by several authors for three compact binary stars are listed in the Table 5 .

It can be seen from the above Table 5 the theoretical results are very close to the observed results.

Table 3. Secular variation of the orbital elements of four compact binary stars per cycle (Revolution).

\begin{tabular}{cccccc}
\hline Compact binary stars & $\Delta a(\mathrm{~cm} / \mathrm{Re})$ & $e / \mathrm{Re}$ & $\Delta \tilde{\omega}(" / \mathrm{Re})$ & $\Delta \varepsilon_{0}\left({ }^{\prime \prime / R e}\right)$ & $\Delta \tau(\mathrm{s} / \mathrm{Re})$ \\
\hline M33 X-7 & 0 & 0 & $16^{\prime \prime} .68$ & $-32^{\prime \prime} .34$ & 11.04 \\
PSR J0737-3039 & 0 & 0 & 17.08 & -29.92 & 0.37 \\
PSR1913+16 & 0 & 0 & 13.45 & -19.75 & 0.78 \\
PSR1543+12 & 0 & 0 & 7.29 & -13.48 & 0.53 \\
\hline
\end{tabular}

[Note] The symbol denotes arc-second: Rev denotes Revolution (cycle).

Table 4. Secular variable rates of the orbital elements of four compact binary stars per year.

\begin{tabular}{|c|c|c|c|c|c|c|c|}
\hline \multirow{2}{*}{$\begin{array}{l}\text { Compact } \\
\text { binary stars }\end{array}$} & \multirow{2}{*}{ ("/yr) } & \multirow{2}{*}{$\dot{e} \quad(1 / \mathrm{yr})$} & \multicolumn{2}{|c|}{$\dot{\tilde{\omega}}$} & \multicolumn{2}{|c|}{$\dot{\varepsilon}_{0}$} & \multirow{2}{*}{$\dot{\tau}(\mathrm{s} / \mathrm{yr})$} \\
\hline & & & ("/yr) & (deg/yr) & ( "I/yr) & (deg/yr) & \\
\hline M33 X-7 & 0 & 0 & $1765^{\prime \prime}$ & 0.49 & $-3424^{\prime \prime}$ & -0.95 & 19.47 \\
\hline PSR J0737-3039 & 0 & 0 & 61016 & 16.95 & -106875 & -29.68 & 18.45 \\
\hline PSR1913+16 & 0 & 0 & 15218 & 4.22 & -22332 & -6.20 & 14.70 \\
\hline PSR1543+12 & 0 & 0 & 6323 & 1.75 & -11703 & -3.25 & 7.66 \\
\hline
\end{tabular}

Table 5. Comparison of the theoretical results with the observable results.

\begin{tabular}{lccc}
\hline $\begin{array}{l}\text { Compact } \\
\text { binary stars }\end{array}$ & $\begin{array}{c}\text { The theoretical } \\
\text { values } \dot{\tilde{\omega}}(\mathrm{deg} / \mathrm{yr})\end{array}$ & $\begin{array}{c}\text { The observable } \\
\text { values } \dot{\tilde{\omega}}(\mathrm{deg} / \mathrm{yr})\end{array}$ & Authors for providing Data \\
\hline PSRJ0737-3039 & 16.95 & 16.90 & $\begin{array}{c}\text { Kramer et al. (2005) [17] } \\
\text { Burgay et al. (2003) [15] }\end{array}$ \\
PSR1913+16 & 4.2260 & 16.88 & Weisberg \& Taylor (2005) [18] \\
PSR1543+12 & 1.7566 & 4.2266 & Kohacki et al. (2003) [16] \\
\hline
\end{tabular}


2) The possibility of observing effects.

In the solar system the advance of perihelion of Mercury may be observed by the recent instrument. We can see from Table 4 that maximal value of the advance of PSRJ0737-3039 is 61016" per year which correspond to 145000 " time value of advance of perihelion of Mercury in solar system. The value of the advance of periastron of compact binary star is largest than that of Mercury in solar system. Therefore the effects of the advance of compact binary stars can be observed too.

We have four conclusions:

a) The compact binary stars are the best objects for studying the post-Newtonian effects on the orbits

b) Although there are no secular variation for the semi-major axis and eccentricity, there is maximal amplitude of the periodic terms for semi-major axis, such as $\left|A_{\max }\right|=1661.80 \mathrm{~km}$.

c) The longitudes of periastron and the mean longitude at epoch exist both secular and periodic variable terms, and the maximal values for $\dot{\omega}$ and $\dot{\varepsilon}_{0}$ arrive at 16.95/yr and -29.68/yr for PSRJ0737-3039 respectively.

d) The longest time of periastron passage is 19.47 minute per year for black hole M33 X-7. This corresponds to over 3 seconds per a day.

\section{References}

[1] Brumberg, V.A. (1972) Relativistic Celestial Mechanics. Nauk, Moscow. (In Russian)

[2] Brumberg, V.A. (1985) Essential Relativistic Celestial Mechanics. Adam Hilger, Bristol.

[3] Rubincam, D.P. (1977) General Relativity and Satellite Orbit: The Motion of a Test Particle in the Schwarzchild Metric. Celestial Mechanics and Dynamical Astrono$m y$, 15, 21-33. https://doi.org/10.1007/BF01229045

[4] Soffel, M.H., Ruder, H. and Schneider, M. (1987) The Two-Body Problem in the (Truncated) PPN Theory. Celestial Mechanics and Dynamical Astronomy, 40, 77-85. https://doi.org/10.1007/BF01232326

[5] Soffel, M.H. (1989) Relativity in Celestial Mechanics, Astronomy and Geodesy. Springer, Heidelberg. https://doi.org/10.1007/978-3-642-73406-9

[6] Iorio, L. (2005) On the Possibility of Measuring the Solar Oblateness and Some Relativistic Effects from Planetary Ranging. $A \& A$, 433, 385-393.

[7] Will, C.M. (1981) Theory of Experiment in Gravitational Physics. Cambridge University Press, London, New York, New Rochelle, Melbourne, Sydney.

[8] Will. C.M. (2006) The Confrontation between General Relativity and Experiment. Living Reviews in Relativity, 9, 5-100. https://doi.org/10.12942/lrr-2006-3

[9] Damour. T. and Deruelle, N. (1985) General Relativistic Celestial Mechanics 1. The Post-Newtonian Motion. Annales de I Institut Henri Poincaré, 43, 107-132.

[10] Damour, T. and Deruelle, N. (1986) General Relativistic Celestial Mechanics 11. The Post-Newtonian Timing Formulas. Annales de PInstitut Henri Poincaré, 44, 263-292.

[11] Schāefer, G. and Wex, N. (1993) Second Post-Newtonian Motion of Compact Bina- 
ries. Physics Letters A, 174, 196-205. https://doi.org/10.1016/0375-9601(93)90758-R

[12] Wex, N. (1995) The Second Post-Newtonian Motion of Compact Binary-Star Systems with Spin. Classical and Quantum Gravity, 12, 983.

https://doi.org/10.1088/0264-9381/12/4/009

[13] Calura, M., et al. (1997) Post-Newtonian Lagrangian Planetary Equation. Physical Review D, 56, 4782-4788. https://doi.org/10.1103/PhysRevD.56.4782

[14] Iorio, L. (2007) The Post-Newtonian Mean Anomaly Advance as Further PostKeplerian Parameter in Pulsar Binary Systems. Astrophys \& Space Scince, 312, 331-335.

[15] Burgay, M., et al. (2003) An Increased Estimate of the Merger Rate of Double Neutron Stars from Observation of a Highly Relativistic System. Nature, 426, 531-533. https://doi.org/10.1038/nature02124

[16] Konacki, M., Wolszczan, A. and Stairs, I.H. (2003) Geodetic Precession and Timing of the Relativistic Binary Pulsars B1543+12 and PSR1913+16. The Astrophysical Journal, 589, 495-502. https://doi.org/10.1086/374418

[17] Kramer, M., et al. (2005) The Double Pulsars-A New Test Relativistic Gravity. In: Rasio, F.A. and Stairs, I.H., Eds., ASP Conference Series, 328, 61-65.

[18] Weisberg, J.M. and Taylor, J.H. (2005) The Relativistic Binary Pulsars B 1913+16. Thirty Years of Observations and Analysis. In: Rasio, F.A. and Stairs, I.H., Eds., $A S P$ Conference Series, 328, 25-31.

[19] Smart, W.M. (1953) Celestial Mechanics. London, New York, Toronto, 19.

[20] Brouwer, D. and Clemence, G.M. (1961) Methods of Celestial Mechanics. Academic Press, New York, London.

[21] Roy, A.E. (1988) Orbital Motion, Adam Hilger, Bristol and Philadelphia, 192.

[22] Willems, B., Kalogera, V. and Henninger, M. (2004) Plusar Licks and Spin Tilts in the Close Double Newtron Stars PSR J0737-3039. PSR B 1534+12 and PSR 1913+16. The Astrophysical Journal, 616, 414-438. https://doi.org/10.1086/424812

[23] Orosz, J.A., et al. (2007) A 15.65-Solar Mass Black Hole in an Eclipsing Binary in the Nearly Spiral Galaxy M33. Nature, 449, 872-875. https://doi.org/10.1038/nature06218

[24] Li, L.-S. (2010) Post-Newtonian Effect on the Variation of Time of Periastron Passage of Binary Stars in Three Gravitational Theories. Astrophysics and Space Science, 327, 59-65. 